\title{
Role of RhoA in Activity-Dependent Cortical Axon Branching
}

\author{
Soichiro Ohnami, ${ }^{1}$ Mitsuharu Endo, ${ }^{2}$ Satoshi Hirai, ${ }^{1}$ Naofumi Uesaka, ${ }^{1}$ Yumiko Hatanaka, ${ }^{1}$ Toshihide Yamashita, ${ }^{2}$ and \\ Nobuhiko Yamamoto ${ }^{1}$ \\ ${ }^{1}$ Laboratory of Cellular and Molecular Neurobiology, Graduate School of Frontier Biosciences, and 2Department of Molecular Neuroscience, Graduate \\ School of Medicine, Osaka University, Suita, Osaka 565-0871, Japan
}

During development, axon branching is influenced by sensory-evoked and spontaneous neural activity. We studied the molecular mechanism that underlies activity-dependent branch formation at horizontally elongating axons (horizontal axons) in the upper cortical layers, focusing on Rho family small GTPases. Axonal labeling with enhanced yellow fluorescent protein showed that horizontal axons formed several branches in organotypic slice cultures. This branch formation was considerably increased by introducing constitutively active RhoA and was slightly inhibited by dominant-negative RhoA. Activators and inhibitors of endogenous RhoA signaling also promoted and inhibited branching, respectively. Daily imaging of horizontal axon growth further demonstrated that constitutively active RhoA increased the dynamic addition and loss of branches. Moreover, the amount of active RhoA relative to the total amount of RhoA was examined by a pull-down assay in cortical slices treated with sodium channel or glutamate receptor blockers to reduce neural activity. Activity blockade significantly decreased active RhoA compared with normal culture conditions, in which spontaneous firing is prominent. These findings suggest that RhoA signaling acts as a positive regulator for activity-dependent axon branching in cortical neurons.

Key words: branch formation; cytoskeleton; RhoA; axon guidance; cerebral cortex; organotypic culture

\section{Introduction}

Axonal branching is an essential process for a neuron connecting with its appropriate target cells in the developing nervous system. To date, some guidance molecules have been demonstrated to regulate axonal branching of central and peripheral neurons (Wang et al., 1999; Ozdinler and Erzurumlu, 2002; Dent et al., 2004). It has also been shown that the size and the complexity of the branches are affected by neural activity (Hubel et al., 1977; Antonini and Stryker, 1993). However, the molecular basis of activity-dependent axonal branching remains mostly unknown.

Pyramidal cells in the upper layers of the neocortex have axons that extend to neurons at some distance within the same layers (horizontal axons). Branch formation in horizontal axons comes about in late developmental stages, in response to the emergence of neural activity (Gilbert and Wiesel, 1989; Callaway and Katz, 1990; Ruthazer and Stryker, 1996). In the present study, we investigate the molecular mechanisms that underlie horizontal

\footnotetext{
Received April 21, 2008; revised July 5, 2008; accepted July 29, 2008.

This work was supported by Grants-in-Aid for Scientific Research Projects 15300107, 18021021, and 18300105 from Japanese Ministry of Education, Culture, Science, and Sports and Novartis Foundation (Japan) for the Promotion of Science. We thank Dr. Granseth for critical reading of this manuscript.

Correspondence should be addressed to Nobuhiko Yamamoto, Laboratory of Cellular and Molecular Neurobiology, Graduate School of Frontier Biosciences, Osaka University, Suita, Osaka 565-0871, Japan. E-mail: nobuhiko@fbs.osaka-u.ac.jp.

S. Ohnami's present address: Shionogi Aburahi Laboratories, Shionogi \& Co. Ltd., Kohka, Shiga 520-3423, Japan

N. Uesaka's present address: Department of Neurophysiology, Graduate School of Medicine, University of Tokyo, 7-3-1, Hongo, Bunkyo-ku, Tokyo, 113-0033, Japan.

Y. Hatanaka's present address: Division of Cell Biology, Graduate School of Biosciences, Nara Institute of Science and Technology, Ikoma, Nara, 630-0192, Japan.

D0I:10.1523/JNEUROSCI.1731-08.2008

Copyright $\odot 2008$ Society for Neuroscience $\quad 0270-6474 / 08 / 289117-05 \$ 15.00 / 0$
}

axon branching, focusing on Rho family small GTPases, because this molecular species can regulate cytoskeletal proteins and may integrate various extracellular signals that modulate axonal branching (Kimura et al., 1996; Luo et al., 1996; Ruchhoeft et al., 1999; Nakayama et al., 2000; Tashiro et al., 2000; Wahl et al., 2000; Yamaguchi et al., 2001; Burridge and Wennerberg, 2004). To address this issue, we used a gene transfer technique in neocortical slice culture preparations accessible to daily imaging, electrophysiology, and biochemical analysis. The result demonstrated that active RhoA was increased by neural activity and promoted branch formation, indicating that RhoA signaling contributes to activity-dependent axon branching of cortical neurons.

\section{Materials and Methods}

Organotypic slice culture. All experiments were performed according to the guidelines laid down by the animal welfare committees of Osaka University. The method for cortical slice cultures has been described previously (Yamamoto et al., 1992; Uesaka et al., 2005). In brief, coronal slices (250 $\mu \mathrm{m}$ thickness) were dissected from the occipital cortex of postnatal day 0 or 1 rat (Sprague Dawley; Nihon-Dobutsu), were cultured on the collagen-coated membranes (Millicell-CM PICMORG50; Millipore) in serum-free, hormone-supplemented medium, and maintained at $37^{\circ} \mathrm{C}$ in an environment of humidified $95 \%$ air and $5 \% \mathrm{CO}_{2}$. To monitor neural activity, cortical slices were plated on multielectrode dishes (MED; Alpha MED Sciences) (Uesaka et al., 2005).

Preparation of plasmids and gene delivery into cultured cells. The coding region of enhanced yellow fluorescent protein (EYFP) was cloned into pCAGGS. Human RhoV14 and RhoN19 (generous gifts from Dr. Kaibuchi, Graduate School of Medicine, Nagoya University, Nagoya, Japan) (Amano et al., 1998) were hemagglutinin epitope (HA) tagged at the $\mathrm{N}$ 
terminal and cloned into a vector with $\mathrm{T} \alpha 1$ promoter, a neuron-specific promoter. Electroporation with glass microelectrodes was used for gene transfer to the cultured cortical cells (Uesaka et al., 2005). In brief, the plasmid solution of EYFP $(1-2 \mu \mathrm{g} / \mu \mathrm{l})$ or mixture of EYFP (1-2 $\mu \mathrm{g} / \mu \mathrm{l})$ plus Rho GTPases $(3-4 \mu \mathrm{g} / \mu \mathrm{l})$ was pressure ejected to the surface of the explants with a glass micropipette. Three to five trains of 200 square pulses ( 1 ms duration, $200 \mathrm{~Hz}, 500-$ $700 \mu \mathrm{A})$ were delivered with another glass micropipette to three or four sites per slice.

Confocal imaging and quantitative analysis. EYFP-labeled axons were observed by confocal microscopy in living conditions at room temperature (MRC-600, 40×, Nikon long-distance objective lens; numerical aperture, 0.55 ). Confocal images were collected at 1 to $5 \mu \mathrm{m}$ steps to obtain the entire axon arbors (2-20 optical sections). Reconstruction of axonal arbors and quantitative analysis were performed with custom-made macros using NIH Image software. Very small buds $(<3 \mu \mathrm{m})$ from the axon shaft were excluded from the analysis. The number of branch points and the lengths of individual branch tip segments were measured. Statistical analysis was performed with MannWhitney $U$ test.

Daily imaging. Labeled axons were observed by confocal microscopy for $\sim 5$ min every $24 \mathrm{~h}$. Branches were classified to five categories as described previously (Uesaka et al., 2005). In brief, branches that grew or retracted $<5 \mu \mathrm{m}$ in length during $24 \mathrm{~h}$ were termed as "stable." Branches that became longer or shorter $>5 \mu \mathrm{m}$ were termed as "grow" or "retract," respectively. Newly added branches were termed as "added.” Disappearing branches were termed as "lost."

Pharmacological treatment. To change endogenous RhoA activity, lysophosphatidic acid (LPA) (30 $\mu \mathrm{M}$; Sigma), a bioactive lipid that activates the RhoA signaling pathway, and Y-27632 (4-[(1R)-1-aminoethyl]-Npyridin-4-yl-cyclohexane-1-carboxamide) ( $50 \mu \mathrm{M}$; a gift from Yoshitomi Pharmaceuticals, Tokyo, Japan), a specific inhibitor of a Rhodependent serine/threonine kinase (ROCK), were added to the culture medium between 1 and 2 weeks in vitro (Li et al., 2000; Nakayama et al., 2000). To suppress neural activity, either tetrodotoxin (TTX) (100 nм; Seikagaku-Kogyo) or D-APV (100 $\mu \mathrm{M}$; Tocris Cookson) plus DNQX (20 $\mu \mathrm{M}$; Tocris Cookson) was added to the medium. These drug-containing media were exchanged every other day (Nakayama et al., 2000; Uesaka et al., 2005; Xu et al., 2007).

Immunohistochemistry. Cortical slices were fixed with $4 \%$ paraformaldehyde overnight at $4^{\circ} \mathrm{C}$. Slices were preincubated in a blocking solution containing 5\% goat (Vector Laboratories) or donkey (Millipore Bioscience Research Reagents) serum and 0.3\% Triton X-100 in PBS for $2 \mathrm{~h}$ at room temperature. Slices were then incubated with anti-HA antibody (1:100; Roche Diagnostics) overnight at $4^{\circ} \mathrm{C}$. After three washes, the slices were incubated in cyanine 3-conjugated goat or donkey anti-mouse secondary antibody (1:500; Jackson ImmunoResearch) for $2 \mathrm{~h}$ at room temperature, followed by extensive washes. The slices were mounted on slide glasses and examined using epifluorescence or confocal microscopy.

Pull-down assay. RhoA activity was measured by pull-down assay using glutathione $S$-transferase (GST) fusion protein of Rho binding domain (RBD). Cortical slices were homogenized in a lysis buffer $(50 \mathrm{~mm}$ HEPES, pH 7.4, 1\% NP-40, 0.5\% sodium deoxycholate, 0.1\% SDS, 5\% glycerol, $150 \mathrm{~mm} \mathrm{NaCl}, 30 \mathrm{~mm} \mathrm{MgCl}_{2}, 10 \mathrm{~mm} \mathrm{NaF}, 1 \mathrm{~mm} \mathrm{Na} \mathrm{VO}_{4}, 10$ $\mu \mathrm{g} / \mathrm{ml}$ aprotinin, and $10 \mu \mathrm{g} / \mathrm{ml}$ leupeptin). Cleared lysates were incubated for $45 \mathrm{~min}$ at $4^{\circ} \mathrm{C}$ with GST-RBD coupled beads $(20-30 \mu \mathrm{g} / \mathrm{sam}$ ple). Precipitates were washed three times with the lysis buffer and sus-

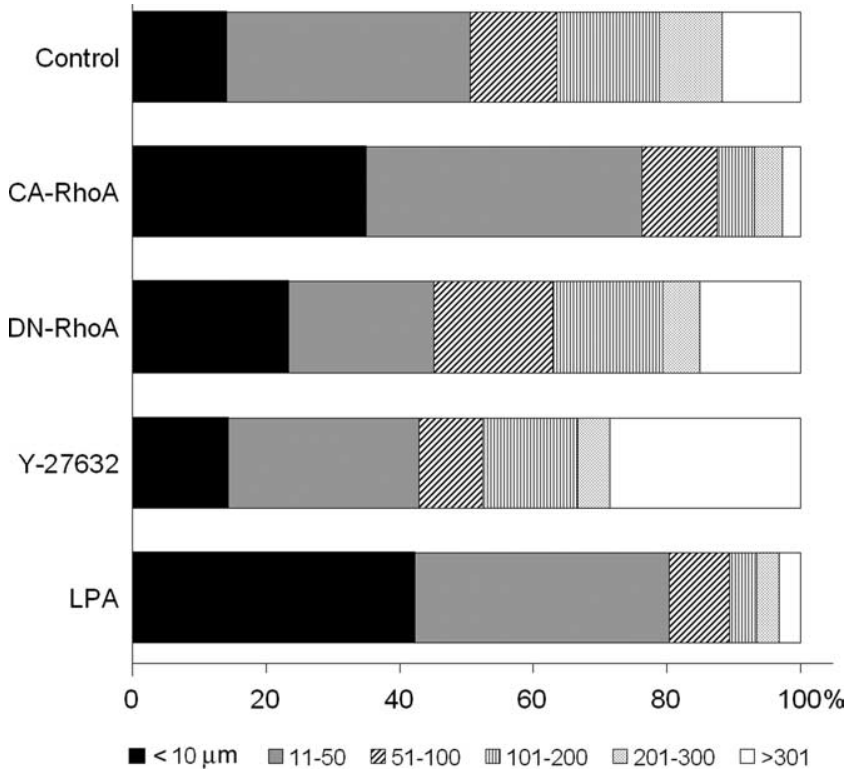

Figure 2. The number of small branches is increased by RhoA. The distribution bar graphs regarding branch length is shown in each case (see Fig. $1 E-I$ ). Note that the population of short branches ( $<50 \mu \mathrm{m}$; black and gray columns) is much larger in CA-RhoA-expressing and LPAtreated horizontal axons.

pended in the SDS sample buffer. Proteins were separated by $12 \%$ SDSPAGE and transferred to polyvinylidene difluoride membrane. GTPbound RhoA and total RhoA were detected by immunoblotting using a monoclonal RhoA antibody (Santa Cruz Biotechnology). 

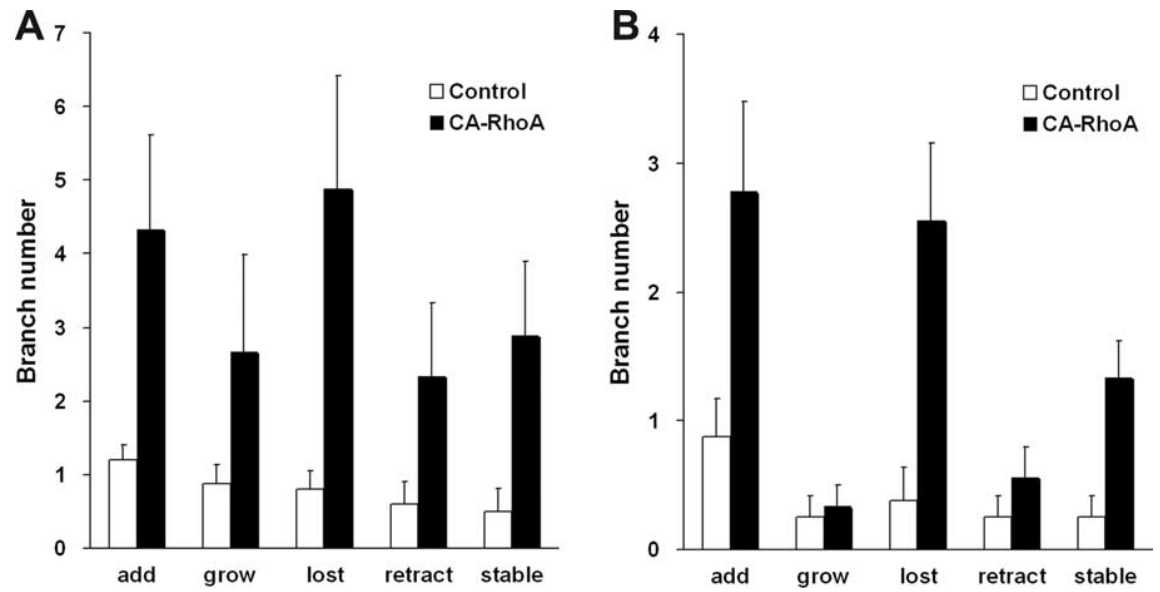

Figure 3. Branch dynamics is increased by RhoA. $\boldsymbol{A}$, Individually distinguishable horizontal axons were followed every day for 3- $6 \mathrm{~d}$. All of the observed branches were classified into five categories. The number of branches (per day) (mean and SEM) in each category was counted for the control axons (open columns) and CA-RhoA-expressing axons (filled columns). B, Similar histogram but only including branches with smaller than $10 \mu \mathrm{m}$.

nique (see Materials and Methods). Individual horizontal axons were subsequently observed by confocal microscopy. To examine the role of RhoA signaling, a plasmid encoding constitutively active (CA) or dominant-negative (DN) RhoA (Amano et al., 1998) was introduced together with the EYFP plasmid. Immunohistochemistry for a peptide tag inserted into CA- and DN-RhoA demonstrated that $>92 \%$ of EYFP-labeled neurons strongly expressed CA- or DN-RhoA (Fig. 1A,B).

Horizontal axons grew along the upper layers in the cortical explants, and, after 2 weeks in culture, several branches formed at a distance of $\sim 1 \mathrm{~mm}$ from the cell body (Fig. 1C,E) (Uesaka et al., 2005). When CA-RhoA was coexpressed with EYFP, the branching of labeled horizontal axons was found to increase dramatically compared with EYFP-expressing controls (Fig.

DNQX Control TTX Control + APV
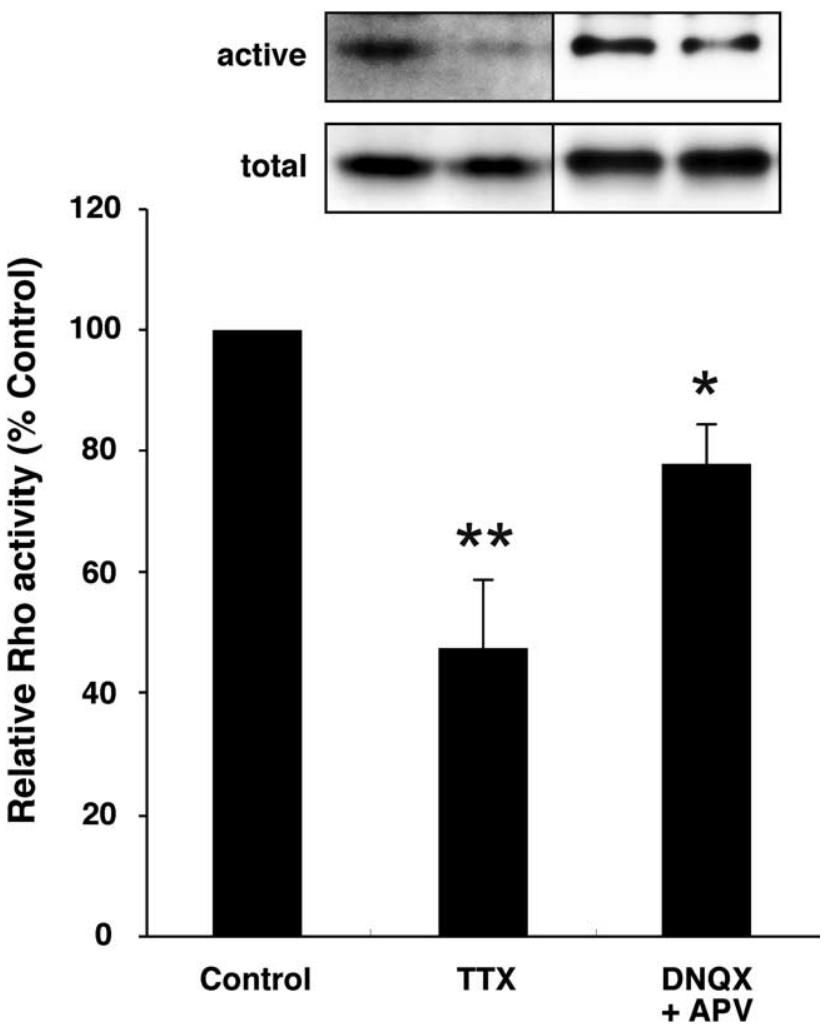

Figure 4. RhoA activity is decreased by blocking neural activity. The amounts of GTP-bound RhoA and total RhoA were detected by the pull-down assay (inset). Relative RhoA activity (GTP-bound RhoA/total RhoA) (mean and SEM) was calculated for control, TTX-treated, and APV/DNQX-treated explants. ${ }^{*} p<0.05 ;{ }^{* *} p<0.01$.

\section{Results}

Branch formation of horizontal axons was studied in organotypic cortical slice cultures, in which laminar cytoarchitecture was preserved (Yamamoto et al., 1992; Uesaka et al., 2005). A small number of upper layer cells in cortical slices were labeled with EYFP by introducing its expression vector using an electroporation tech-
$1 D, F)$. Quantitative analysis (Fig. $1 \mathrm{~J}$ ) showed that the number of branch points was approximately three times larger in CA-RhoAexpressing axons $(14.9 \pm 2.9 ; n=19 ; p<0.01)$ than in the control $(4.7 \pm 1.4 ; n=15)$. In DN-RhoA-expressing axons, the average number of branch points was slightly decreased (3.4 \pm $0.6 ; n=15$ ) (Fig. 1G,J), although this difference was not statistically significant. To examine endogenous RhoA activity, a ROCK inhibitor $(\mathrm{Y}-27632,50 \mu \mathrm{M})$ was added to the culture medium between 1 and 2 weeks in culture, when most branches emerge from horizontal axons in vivo and in vitro (Uesaka et al., 2005). The number of branching points was considerably decreased in the presence of Y-27632 $(1.3 \pm 0.5 ; n=9 ; p<0.05)($ Fig. $1 H, J)$. Conversely, application of LPA $(30 \mu \mathrm{M})$, a RhoA activator, markedly increased the number of branching points $(14.6 \pm 2.8 ; n=8$; $p<0.01$ ) (Fig. 1I,J), similar to what was found for CA-RhoAexpressing axons. Thus, the results obtained by the pharmacological treatments that affect all cultured cells were consistent with those in CA- and DN-RhoA experiments that were specific for individual cells. Moreover, the LPA-induced increase in branching was diminished after introducing DN-RhoA to the cells (data not shown), indicating that the branch-promoting activity of LPA is mediated by activation of the endogenous RhoA pathway. Together, these findings suggest that branch formation in horizontal axons is promoted by RhoA activation.

A characteristic aspect in horizontal axon branching by RhoA activation was the emergence of small branches. Quantitative analysis of branch length clearly demonstrated that the number of short branches $(<50 \mu \mathrm{m})$ was much increased with CA-RhoA and LPA application (Fig. 2). On the contrary, the fraction of short branches was slightly decreased with DN-RhoA and Y-27632 application. To examine how short branches emerge by RhoA activation, daily imaging was performed. The result showed that the numbers of elongating ("add" and "grow"), eliminating ("lost" and "retract"), and stable branches were dramatically increased in CA-RhoA-expressing axons (Fig. 3). In particular, most of the smallest ( $\leq 10 \mu \mathrm{m}$ ) branches in CA-RhoAexpressing axons were found to be classified as add or lost (Fig. 3). These findings indicate that RhoA promotes dynamics of horizontal axon branching.

Our previous study has shown that neural activity is required for horizontal axon branching (Uesaka et al., 2005). Horizontal axon branching was substantially suppressed in medium con- 
taining the sodium channel blocker TTX (100 nM) or the glutamate receptor blockers DNQX and APV (20 and $100 \mu \mathrm{M}$, respectively), which abolished the firing activity (supplemental Fig. 1, available at www.jneurosci.org as supplemental material) (Uesaka et al., 2005). An interesting hypothesis is that RhoA may be involved in such an activity-dependent process. In agreement with this view, axonal branching was promoted in CA-RhoAexpressing axons even in the presence of TTX (supplemental Fig. 2 , available at www.jneurosci.org as supplemental material).

To further study whether RhoA mediates neural activity, a pull-down assay for endogenous active RhoA was performed in the cortical slice cultures that had been subjected to pharmacological treatments with either TTX or DNQX and APV. As a result, the ratio of the amount of active RhoA to the total RhoA was much decreased in the TTX-treated cultures (Fig. 4). A significant decrease of active RhoA was also found in cultured slices treated with DNQX and APV (Fig. 4). The pull-down assay was further performed in LPA-treated cultures to examine the fully activated state. After the chronic LPA treatment, however, there were no apparent changes in active RhoA (data not shown) (Ren et al., 1999). Transient RhoA activation might be sufficient for increasing small branches in the horizontal axons.

\section{Discussion}

The present results demonstrate that active RhoA promotes horizontal axon branching and is increased by neural activity, indicating that RhoA acts as a mediator for activity-dependent branch formation. Previously, neural activity has been shown to regulate dendritic branching by altering RhoA activity (Li et al., 2000; Sin et al., 2002). To our knowledge, we are the first to demonstrate the importance of RhoA signaling in activitydependent axon branching. However, it is unlikely that RhoA is the only regulator, because active RhoA increased the number of small branches, whereas larger branches were unaffected. Considering the result from the daily imaging study, RhoA might be more important for the dynamic aspect of branch formation. Moreover, the fact that TTX application reduced the amount of active RhoA more efficiently than the application of glutamate receptor antagonists suggests that RhoA activation may be more sensitive to firing activity than to synaptic activity.

RhoA activation has been shown to inhibit dendritic formation and neuritic outgrowth in various neuronal types (Bito et al., 2000; Li et al., 2000; Nakayama et al., 2000; Wong et al., 2000). Conversely, growth-promoting effects have been reported for cortical and hippocampal cell axons (Threadgill et al., 1997; Ahnert-Hilger et al., 2004), consistent with our present result. Rac, another Rho family small GTPase, also affects axonal growth and guidance ( $\mathrm{Ng}$ et al., 2002). We examined the action of Racl in cortical axon branching using DN and CA forms, but no apparent effects were observed in upper layer cell axons (data not shown). Thus, Rho GTPases may regulate cytoskeleton organization by means of different downstream molecules, depending on cell type and developmental stage.

It has been shown that Rho GTPases are involved in activitydependent spine formation and dendritic arborization ( $\mathrm{Li}$ et al., 2000; Sin et al., 2002; Schubert et al., 2006; Nakazawa et al., 2008). Several guanine nucleotide exchange factors and GTPaseactivating proteins (GAPs), including p250GAP, Lfc, and $\beta$ PIX, which are expressed in the mammalian neocortex, regulate dendritic morphology in response to neural activity (Ryan et al., 2005; Nakazawa et al., 2008; Saneyoshi et al., 2008). Similar mechanisms might work in axons, although additional investigation is necessary because the role of Rho GTPases in activity- dependent axonal branching has not been realized until the present study.

RhoA has been demonstrated to act in signaling pathways triggered by guidance molecules or trophic factors (Jin and Strittmatter, 1997; Yamashita et al., 1999; Wahl et al., 2000; Yamaguchi et al., 2001). The present findings strongly suggest that RhoA transduces activity-dependent signals as well. Thus, RhoA could contribute to the regulation of axonal branching by integrating chemical guidance cues and neural activity signals.

\section{References}

Ahnert-Hilger G, Höltje M, Grosse G, Pickert G, Mucke C, NixdorfBergweiler B, Boquet P, Hofmann F, Just I (2004) Differential effects of Rho GTPases on axonal and dendritic development in hippocampal neurones. J Neurochem 90:9-18.

Amano M, Chihara K, Nakamura N, Fukata Y, Yano T, Shibata M, Ikebe M, Kaibuchi K (1998) Myosin II activation promotes neurite retraction during the action of Rho and Rho-kinase. Genes Cells 3:177-188.

Antonini A, Stryker MP (1993) Rapid remodeling of axonal arbors in the visual cortex. Science 260:1819-1821.

Bito H, Furuyashiki T, Ishihara H, Shibasaki Y, Ohashi K, Mizuno K, Maekawa M, Ishizaki T, Narumiya S (2000) A critical role for a Rhoassociated kinase, p160ROCK, in determining axon outgrowth in mammalian CNS neurons. Neuron 26:431-441.

Burridge K, Wennerberg K (2004) Rho and Rac take center stage. Cell 116:167-179

Callaway EM, Katz LC (1990) Emergence and refinement of clustered horizontal connections in cat striate cortex. J Neurosci 10:1134-1153.

Dent EW, Barnes AM, Tang F, Kalil K (2004) Netrin-1 and semaphorin 3A promote or inhibit cortical axon branching, respectively, by reorganization of the cytoskeleton. J Neurosci 24:3002-3012.

Gilbert CD, Wiesel TN (1989) Columnar specificity of intrinsic horizontal and corticocortical connections in cat visual cortex. J Neurosci 9:2432-2442.

Hubel DH, Wiesel TN, LeVay S (1977) Plasticity of ocular dominance columns in monkey striate cortex. Philos Trans R Soc Lond B Biol Sci 278:377-409.

Jin Z, Strittmatter SM (1997) Racl mediates collapsin-1-induced growth cone collapse. J Neurosci 17:6256-6263.

Kimura K, Ito M, Amano M, Chihara K, Fukata Y, Nakafuku M, Yamamori B, Feng J, Nakano T, Okawa K, Iwamatsu A, Kaibuchi K (1996) Regulation of myosin phosphatase by Rho and Rho-associated kinase (Rho-kinase). Science 273:245-248.

Li Z, Van Aelst L, Cline HT (2000) Rho GTPases regulate distinct aspects of dendritic arbor growth in Xenopus central neurons in vivo. Nat Neurosci 3:217-225

Luo L, Hensch TK, Ackerman L, Barbel S, Jan LY, Jan YN (1996) Differential effects of the Rac GTPase on Purkinje cell axons and dendritic trunks and spines. Nature 379:837-840.

Nakayama AY, Harms MB, Luo L (2000) Small GTPases Rac and Rho in the maintenance of dendritic spines and branches in hippocampal pyramidal neurons. J Neurosci 20:5329-5338.

Nakazawa T, Kuriu T, Tezuka T, Umemori H, Okabe S, Yamamoto T (2008) Regulation of dendritic spine morphology by an NMDA receptorassociated Rho GTPase-activating protein, p250GAP. J Neurochem 105:1384-1393.

Ng J, Nardine T, Harms M, Tzu J, Goldstein A, Sun Y, Dietzl G, Dickson BJ, Luo L (2002) Rac GTPases control axon growth, guidance and branching. Nature 416:442-447.

Ozdinler PH, Erzurumlu RS (2002) Slit2, a branching-arborization factor for sensory axons in the mammalian CNS. J Neurosci 22:4540-4549.

Ren XD, Kiosses WB, Schwartz MA (1999) Regulation of the small GTPbinding protein Rho by cell adhesion and the cytoskeleton. EMBO J 18:578-585.

Ruchhoeft ML, Ohnuma S, McNeill L, Holt CE, Harris WA (1999) The neuronal architecture of Xenopus retinal ganglion cells is sculpted by rhofamily GTPases in vivo. J Neurosci 19:8454-8463.

Ruthazer ES, Stryker MP (1996) The role of activity in the development of long-range horizontal connections in area 17 of the ferret. J Neurosci 16:7253-7269.

Ryan XP, Alldritt J, Svenningsson P, Allen PB, Wu GY, Nairn AC, Greengard 
P (2005) The Rho-specific GEF Lfc interacts with neurabin and spinophilin to regulate dendritic spine morphology. Neuron 47:85-100.

Saneyoshi T, Wayman G, Fortin D, Davare M, Hoshi N, Nozaki N, Natsume T, Soderling TR (2008) Activity-dependent synaptogenesis: regulation by a CaM-kinase kinase/CaM-kinase I/betaPIX signaling complex. Neuron 57:94-107.

Schubert V, Da Silva JS, Dotti CG (2006) Localized recruitment and activation of RhoA underlies dendritic spine morphology in a glutamate receptor-dependent manner. J Cell Biol 172:453-467.

Sin WC, Haas K, Ruthazer ES, Cline HT (2002) Dendrite growth increased by visual activity requires NMDA receptor and Rho GTPases. Nature 419:475-480.

Tashiro A, Minden A, Yuste R (2000) Regulation of dendritic spine morphology by the rho family of small GTPases: antagonistic roles of Rac and Rho. Cereb Cortex 10:927-938.

Threadgill R, Bobb K, Ghosh A (1997) Regulation of dendritic growth and remodeling by Rho, Rac, and Cdc42. Neuron 19:625-634.

Uesaka N, Hirai S, Maruyama T, Ruthazer ES, Yamamoto N (2005) Activity dependence of cortical axon branch formation: a morphological and electrophysiological study using organotypic slice cultures. J Neurosci 25:1-9.

Wahl S, Barth H, Ciossek T, Aktories K, Mueller BK (2000) Ephrin-A5 in- duces collapse of growth cones by activating Rho and Rho kinase. J Cell Biol 149:263-270.

Wang KH, Brose K, Arnott D, Kidd T, Goodman CS, Henzel W, TessierLavigne M (1999) Biochemical purification of a mammalian slit protein as a positive regulator of sensory axon elongation and branching. Cell 96:771-784.

Wong WT, Faulkner-Jones BE, Sanes JR, Wong RO (2000) Rapid dendritic remodeling in the developing retina: dependence on neurotransmission and reciprocal regulation by Rac and Rho. J Neurosci 20:5024-5036.

Xu KP, Yin J, Yu FS (2007) Lysophosphatidic acid promoting corneal epithelial wound healing by transactivation of epidermal growth factor receptor. Invest Ophthalmol Vis Sci 48:636-643.

Yamaguchi Y, Katoh H, Yasui H, Mori K, Negishi M (2001) RhoA inhibits the nerve growth factor-induced Racl activation through Rho-associated kinase-dependent pathway. J Biol Chem 276:18977-18983.

Yamamoto N, Yamada K, Kurotani T, Toyama K (1992) Laminar specificity of extrinsic cortical connections studied in coculture preparations. Neuron 9:217-228.

Yamashita T, Tucker KL, Barde YA (1999) Neurotrophin binding to the p75 receptor modulates Rho activity and axonal outgrowth. Neuron 24:585593. 